ARGONNE NATIONAL LABORATORY

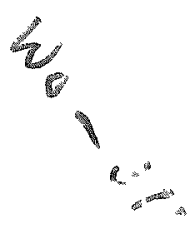

P. O. Box 299

Lemont, IIlinois

THE STRUCTURE OF THE PERRHENATE ION

by

Howard H. Claassen and A. J. Zielen

CHEMISTRY DIVISION

September 8, 1953

Operated by The University of Chicago

under

Contract $W-31-109-$ eng $=38$ 


\section{DISCLAIMER}

This report was prepared as an account of work sponsored by an agency of the United States Government. Neither the United States Government nor any agency Thereof, nor any of their employees, makes any warranty, express or implied, or assumes any legal liability or responsibility for the accuracy, completeness, or usefulness of any information, apparatus, product, or process disclosed, or represents that its use would not infringe privately owned rights. Reference herein to any specific commercial product, process, or service by trade name, trademark, manufacturer, or otherwise does not necessarily constitute or imply its endorsement, recommendation, or favoring by the United States Government or any agency thereof. The views and opinions of authors expressed herein do not necessarily state or reflect those of the United States Government or any agency thereof. 


\section{DISCLAIMER}

Portions of this document may be illegible in electronic image products. Images are produced from the best available original document. 


\title{
THE STRUCTURE OF THE PERRHENATE ION
}

by

Howard H. Claassen* and A. J. Zielen

\begin{abstract}
Raman and infrared spectra have been obtained for aqueous solutions of perrhenic acid and sodium perrhenate. The spectra are interpreted satisfactorily with the assumption that the perrhenate ion in dilute solutions has the tetrahedral symmetry, $\mathrm{ReO}_{4}^{-}$.
\end{abstract}

\section{INTRODUCTION}

The Raman spectra of solutions of perrhenic acid and sodium perrhenate, and of crystalline sodium and potassium perthenate, have been investigated by Fonteyne. 1 He observed only three Raman bands for dilute perrhenic acid and for solutions of sodium perrhenate and concluded that the anion in solution may be $\mathrm{ReO}_{6}^{5}$ with an octahedral structure, rather than $\mathrm{ReO}_{4}^{-}$with a tetrahedral structure as the ion exists in alkali perrhenate crystals. 2 After a study of the ultraviolet absorption spectra of perrienate solution's, as well as some of the chemical properties, J. C. Hindman suggested that the Raman spectra should be reinvestigated.

\section{MATERIALS AND EXPERIMENTAL PROCEDURE}

Rhenium heptaoxide was obtained from the Department of Chemistry of the University of Tennessee. Spectrochemical analys is ${ }^{3}$ of a sample of this material did not reveal the presence of any impurities. Stock solutions of perrhenic acid were prepared by dissolving the heptaoxide in doubly dis tilled water. Weight analyses of the $\mathrm{HReO}_{4}$ content were carried out by titration with standard sodium hydroxide to the methyl red end point. Utilizing a Cary Recording Spectrophotometer, absorption spectra of diluted

*Department of Physics, Wheaton College, Wheaton, Illinois. Participating Institution Appointment.

${ }^{1}$. Fonteyne, Natuurw. Tijdschr. Belg. 20, 20 (1938).

${ }^{2}$ J. Beintema, Z. Krist. $97 A, 300$ (1937).

${ }^{3}$ Kindly performed by J. A. Goleb of this Laboratory. 
aliquots of the perrhenic acid stock solutions were obtained. These did not indicate the presence of any lower oxidation states of rhenium and were in quantitative agreement with the results reported by Hindman and Wehner.4

The sodium perrhenate solution was prepared by adding a slight excess of perrhenic acid to $C P$ grade sodium hydroxide pellets. The pH of the solution was then adjusted to 6 by the addition of dilute sodium hydroxide.

A Hilger E612 Raman Spectrograph, having two $63^{\circ}$ glass prisms, camera aperture $F / 5.7$, and inverse dispersion on the plate of $16 \AA$ per $\mathrm{mm}$ at $4358 \AA$, was used. The Raman excitation unit was obtained from Applied Research Laboratories; it has been described by Kemp, Jones, and Durkee. 5 A $21 \mathrm{ml}$ Raman tube was used. It was filled by forcing the solutions through fine fritted glass filters by means of compressed air.

Raman spectra were recorded on Kodak $103 \mathrm{a}-\mathrm{J}$ and III-J plates. Sodium nitrite solutions were used as light filters. Frequencies were determined by linear interpolation between nearby lines on an adjacent iron arc spectrum. Measurements were made using a Gaertner Mode1 M1205C comparator modified by the addition of the photoelectric setting device described by Tomkins and Fred.6 A Leeds and Northrup photoelectric microphotometer was used to trace some of the spectra. Qualitative information about the state of polarization of Raman bands was obtained by taking two equal exposures with polaroid sheets around the Raman tube, one transmitting the electric vector perpendicular to and one parallel to the axis of the tube.

Infrared spectra were obtained by means of a Model 21 Perkin-Elmer Spectrophotometer with $\mathrm{NaCl}$ prism. Silver chloride windows $0.25 \mathrm{~mm}$ thick and coated with silver sulfide were used to contain the sample. A drop of solution was placed on one window, the other window was laid on, and they were held pressed together with thumb and finger and clamped between brass collars.

\section{RESULTS AND DISCUSSION}

Raman spectra were obtained for six different concentrations of perrhenic acid solutions and for one of sodium perrhenate. For all except the most concentrated acid only three Raman shifts were observed in

4 J. C. Hindman and P. Wehner, J. Am. Chem. Soc. 75, 2869 (1953).

${ }^{5}$ Kemp, Jones, and Durkee, J. Opt. Soc. Am. 42, 811 (1952).

${ }^{6}$ F. S. Tomkins and M. Fred, J. Opt. Soc. Am. 41, 641 (1951). 
addition to the diffuse scattering due to the water. The averages of the measured frequency shifts and the Kohlrausch designations of the exciting Hg lines for which each shift was observed are given in Table I. The relative intensities and widths of the bands may be estimated from Figure $1 \mathrm{~A}$, a photograph of a microphotometer trace showing the three shifts from $4358 \AA$ for the $70 \%$ perrhenic acid solution. The spectra for the more dilute solutions are es sentially identical to this one. For the most concentrated solution, however, several new bands were observed. These are shown, and their frequencies listed, in Figure $1 \mathrm{~B}$. An additional weak band, whose assignment must be considered doubtful, was observed shifted $1612 \mathrm{~cm}^{-1}$ from $4358 \AA$.

Table I

RAMAN FREQUENCIES AND THE EXCITING Hg LINES FOR WHICH EACH WAS OBSERVED, FOR VARIOUS PERRHENATE SOLUTIONS

\begin{tabular}{|l|l|l|l|}
\hline $\mathrm{NaReO}_{4}, 25.4 \%$ & $331 \pm \mathrm{e}, \mathrm{k}$ & $920 \mathrm{e}, \mathrm{k}, \mathrm{i}$ & $970.7 \mathrm{e,k,i}$ \\
$\mathrm{HReO}_{4}, 6 \%$ & $331 \pm \mathrm{e}, \mathrm{k}$ & $919 \mathrm{e}, \mathrm{k}$ & $970.3 \pm \mathrm{e}, \mathrm{k,i}$ \\
$\mathrm{HReO}_{4}, \quad 10.0 \%$ & $331 \pm \mathrm{e}, \mathrm{k}$ & $920 \mathrm{e}, \mathrm{k}$ & $970.7 \pm \mathrm{e}, \mathrm{k}, \mathrm{i}$ \\
$\mathrm{HReO}_{4}, 28.5 \%$ & $332 \pm \mathrm{e}, \mathrm{k}, \mathrm{i}$ & $918 \mathrm{e}, \mathrm{k}, \mathrm{i}$ & $970.7 \pm \mathrm{e}, \mathrm{k}, \mathrm{i}$ \\
$\mathrm{HReO}_{4}, 70.0 \%$ & $332 \pm \mathrm{e}, \mathrm{k}, \mathrm{i}$ & $916 \mathrm{e}, \mathrm{k,i}$ & $971.8 \pm \mathrm{e}, \mathrm{k}, \mathrm{i}, 1$ \\
$\mathrm{HReO}_{4}, 80.1 \%$ & $333 \pm \mathrm{e}$ & $916 \mathrm{e}$ & $974.8 \mathrm{e,k}$ \\
\hline
\end{tabular}

The great difference in exposure times for Figures $1 \mathrm{~A}$ and $1 \mathrm{~B}$ was necessary because of absorption in the $87.7 \%$ sample. This solution appeared yellowish green; the $80.1 \%$ solution appeared slightly yellowish, but all the more dilute solutions were colorless. Because of the absorption, the relative intensity of the $338 \mathrm{~cm}^{-1}$ band is probably considerably greater than indicated in Figure $1 \mathrm{~B}$.

Polarization exposures were made on a $10 \% \mathrm{HReO}_{4}$ solution. These showed the $971 \mathrm{~cm}^{-1}$ band to be polarized and indicated the other two to be depolarized. This is as one would expect by noting the high intensity and sharpness of the $971 \mathrm{~cm}^{-1}$ band.

The infrared spectrum was scanned from 700 to $1400 \mathrm{~cm}^{-1}$ for the sodium perrhenate solution and for three different concentrations of per rhenic acid. Only one absorption maximum due to the perrhenate ion was found in each case, a strong band at $914 \mathrm{~cm}^{-1}$.

The data of Eonteynel agree well with the present Raman data for dilute solutions, but some differences may be noted for the most concentrated solutions. Thus he lists a band at $1102 \mathrm{~cm}^{-1}$ which we did not observe, and we find bands at 886 and $989 \mathrm{~cm}^{-1}$ which he apparently did not resolve from 
the neighboring stronger bands. More puzzling is the disagreement in regard to the concentrations at which extra bands may be observed. Fonteyne lists a band at $840 \mathrm{~cm}^{-1}$ for $30 \% \mathrm{HReO}_{4}$, whereas we found the corresponding band only at $87.7 \%$ concentration. For example, in the case of the $70 \%$ solution, exposures 30 times as great as the one shown in Figure $1 \mathrm{~A}$ showed no evidence of this band.

\section{STRUCTURE CONSIDERATIONS}

Fonteyne ${ }^{1}$ has suggested that the perrhenate ion in dilute solution may be octahedral rather than tetrahedral. The number of fundamental vibrations of each symmetry species and the selection rules for their observation are listed in Table II both for $\mathrm{XY}_{4}$ molecules of $\mathrm{T}_{\mathrm{d}}$ symmetry and $\mathrm{XY}_{6}$ molecules of $O_{h}$ symmetry. Fonteynel believed that only three Raman bands could be ascribed to the ion, and that therefore the $O_{h}$ structure was most plausible. When the absence of a fourth Raman band, except in very concentrated solutions, was verified in the present work, an infrared investigation was attempted. The Raman band at $971 \mathrm{~cm}^{-1}$, since it is polarized, sharp, and intense, must be due to a totally symmetric vibration, and would be observable by absorption neither for the $T_{d}$ nor for the $O_{h}$ structures. The band at about $918 \mathrm{~cm}^{-1}$, on the other hand, must belong to species $F_{2}$ and be infrared active if the symmetry is $T_{d}$, but cannot be infrared active if it is $O_{h}$, since for that symmetry no vibrational mode can be active in both infrared and Raman spectra. Fortunately, water has no strong absorption bands in this region, and it was possible to observe an infrared band at $914 \mathrm{~cm}^{-1}$ due to the perrhenate ion. This is so close to the observed $918 \mathrm{~cm}^{-1}$ Raman band that it is quite plausible they are both due to the same vibrational mode.

The experimental data leave two choices: (1) The symmetry is $\mathrm{T}_{\mathrm{d}}$ and the reason one does not observe four $R$-bands is that the $E$ and one of the $\mathrm{F}_{2}$ vibrations happen to lie so close together that one cannot resolve them, or (2) The symmetry is $O_{h}$ and the close agreement between the one Raman and the infrared band is accidental; they are actually due to vibrational modes of different species. Either choice requires the assumption of an accidental coincidence, the first perhaps more probable since the observed Raman band at $331 \mathrm{~cm}^{-1}$ is quite broad, as shown in Figure 1.

Further light on this question may be obtained by calculation. Heath and Linnett ${ }^{7}$ have shown that for several types and many individual molecules and anions it is possible to calculate ir equencies to within one or two per cent,

${ }^{7}$ D. F. Heath and J.W. Linnett, Trans. Faraday Soc. 44, 873, 878, 884 $(1948): 45,264(1949)$. 
using an intramolecular force field between bonded atoms in accordance with modern theories of directed valency plus an assumed Van der Waals type of repulsion between non-bonded atoms. They have given equations both for tetrahedral $\mathrm{XY}_{4}$ and for octahedral $\mathrm{X} \mathrm{Y}_{6}$ types of molecules. In either case the vibration frequencies for one molecule are given in terms of three potential constants. These equations have been used for the present problem.

Table II

SELECTION RULES FOR THE TWO MODELS CONSIDERED

\begin{tabular}{|c|c|c|c|c|}
\hline \multirow{2}{*}{$\begin{array}{l}\text { Symmetry } \\
\text { Species }\end{array}$} & \multirow{2}{*}{$\begin{array}{c}\text { Number } \\
\text { of } \\
\text { Vibrations }\end{array}$} & \multicolumn{2}{|c|}{ Selection Rules } & \multirow{2}{*}{ Assignment } \\
\hline & & Raman & Infrared & \\
\hline \multicolumn{5}{|c|}{$\mathrm{T}_{\mathrm{d}}$ symmetry, $\mathrm{ReO}_{4}^{-\infty}$} \\
\hline $\mathbf{A}_{1}$ & 1 & Allowed & Forbidden & 971 \\
\hline $\mathbf{E}$ & 1 & Allowed & Forbidden & 331 \\
\hline$F_{2}$ & 2 & Allowed & Allowed & 918,331 \\
\hline \multicolumn{5}{|c|}{$\mathrm{O}_{\mathrm{h}}$ symmetry, ReO } \\
\hline$A_{1 g}$ & 1 & Allowed & Forbidden & $(971)$ \\
\hline$E_{g}$ & 1 & Allowed & Forbidden & $(918)$ \\
\hline$F_{\mathrm{Zg}}$ & 1 & Allowed & Forbidden & (331) \\
\hline$F_{\text {Zu }}$ & 2 & Forbidden & Allowed & - \\
\hline$F_{1}$ & 1 & Forbidden & Forbidden & $=$ \\
\hline
\end{tabular}

First, assuming an $O_{h}$ ion, $R e O_{6}^{5-}$, the three observed Raman bands at 971,918 , and $331 \mathrm{~cm}^{-1}$ were assigned to species $A_{1}$, $E_{g}$, and $F_{2 g}$, respectively, and three potential constants evaluated: $k_{1}=7.59, k_{\beta}=0.337$ and $A=0.162 \times 10^{5}$ dynes/cm. Then the two frequencies of species $F_{1}$, which should be infrared active, were calculated. The result was 315 and $1000 \mathrm{~cm}^{-1}$.

Second, assuming a $T_{d}$ ion, $\mathrm{ReO}_{4}^{-}$, the Raman bands at 971,918 , and $331 \mathrm{~cm}^{-1}$ were assigned to $A_{1}, F_{2}$, and $E_{2}$, respectively, and three potential constants evaluated: $k_{1}=6.52, k_{\alpha}^{\prime}=0.400, A=0.295 \times 10^{5}$ dynes $/ \mathrm{cm}$. The other $\mathrm{F}_{2}$ frequency could then be calculated. The result was $343 \mathrm{~cm}^{-1}$. 
These calculations seem definitely to favor the tetrahedral assumption. The fourth frequency calculated for this assumption is only $12 \mathrm{~cm}^{-1}$ from the observed peak at $331 \mathrm{~cm}^{-1}$, so that it is very plausible that this observed Raman band at $331 \mathrm{~cm}^{-1}$ is a superposition of two bands too close to resolve, particularly in view of the width of the observed band. On the other hand, the octahedral assumption leads to a calculated value for the high infrared frequency of $1000 \mathrm{~cm}^{-1}$, which is $86 \mathrm{~cm}^{-1}$ or $9 \%$ from the observed band at $914 \mathrm{~cm}^{-1}$. This deviation between calculated and observed is much greater than Heath and Linnett ${ }^{7}$ found in any of their examples.

On the basis of the spectroscopic evidence, then, it seems most likely that the perrhenate ion in dilute solution is tetrahedral. The source of the additional Raman bands and of the green color in concentrated solutions may well be the undissociated acid, $\mathrm{HORO}_{3}$. The difference in Raman spectra of dilute and highly concentrated perchloric acid presents a very similar case. 8,9

\section{ACKNOWLEDGMENTS}

The authors are indebted to Dr. J. C. Hindman for suggesting the problem and to Dr. Sheffield Gordon for making available the infrared spectrophotometer.

${ }^{8}$ J. H. Hibben, The Raman Effect and its Chemical Applications (Rheinhold Publishing Corp., New York, 1939) p. 376.

${ }^{9}$ Redlich, Holt, and Bigeleisen, J. Am. Chem. Soc, 66, 13 (1944). 

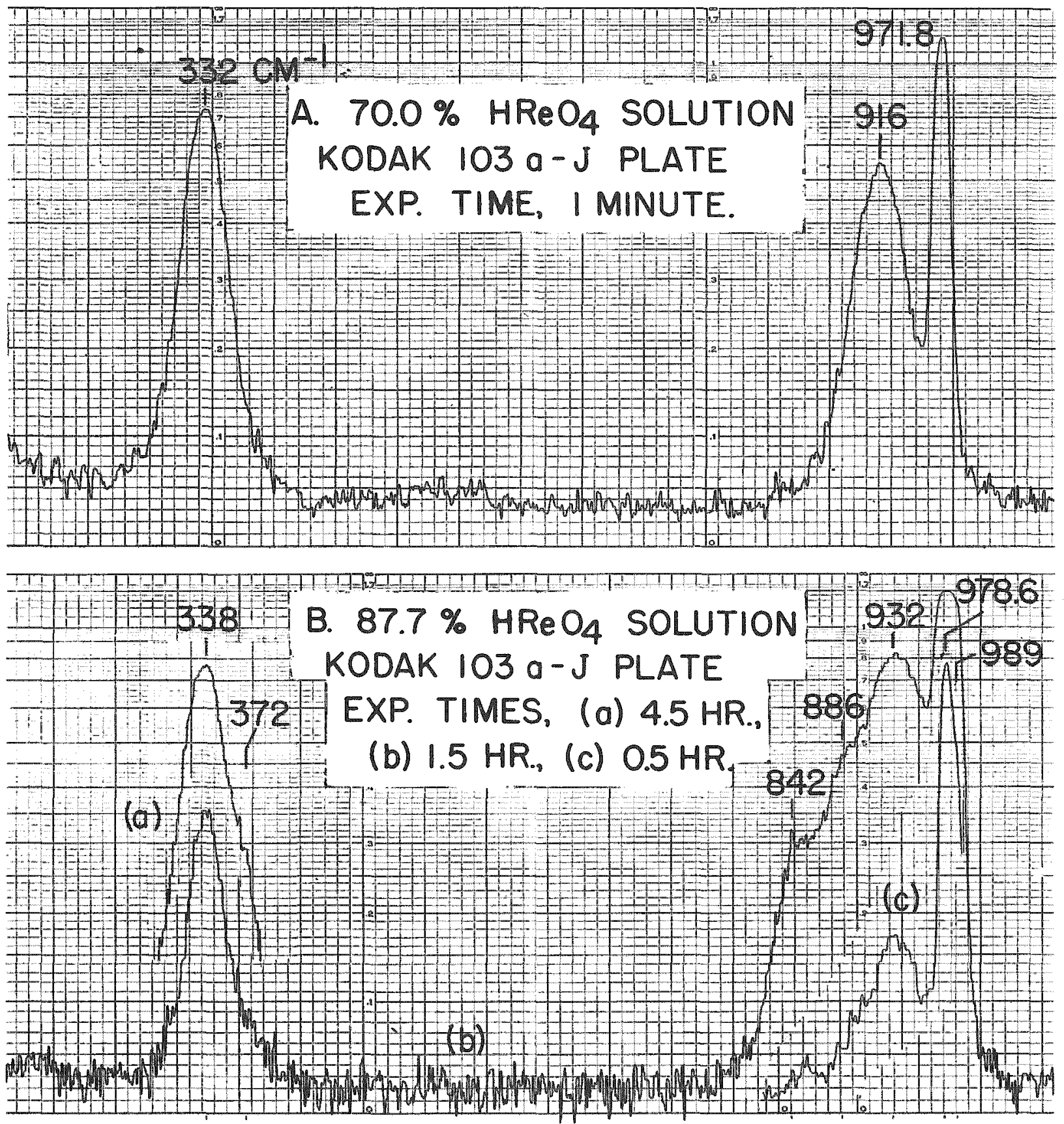

FIGURE I.

MICROPHOTOMETER TRACES OF RAMAN SPECTRA. 